\title{
Orlistat Treatment of Unconjugated Hyperbilirubinemia in Crigler-Najjar Disease: A Randomized Controlled Trial
}

\author{
ANJA M. HAFKAMP, RINEKE NELISSE-HAAK, MAARTEN SINAASAPPEL, RONALD P.J. OUDE ELFERINK, \\ AND HENKJAN J. VERKADE
}

\begin{abstract}
Department of Pediatrics [A.M.H., H.J.V.], Pediatric Gastroenterology, Center for Liver, Digestive and Metabolic Diseases, University of Groningen, 9700 RB, Groningen, The Netherlands; Department of Pediatrics [R.N.-H., M.S.], Sophia Children's Hospital, 3000 CB,

Rotterdam, The Netherlands; Amsterdam Liver Center [R.P.J.O.E.], Academic Medical Center, 1100 DD, Amsterdam, The Netherlands
\end{abstract}

\begin{abstract}
Unconjugated hyperbilirubinemia in Crigler-Najjar (CN) disease is conventionally treated with phototherapy and phenobarbital. Orlistat treatment increases fecal fat excretion and decreases plasma unconjugated bilirubin (UCB) concentrations in Gunn rats, the animal model for $\mathrm{CN}$ disease. We determined in $\mathrm{CN}$ patients the effects of orlistat treatment on plasma UCB concentrations, and on fecal excretion of fat and UCB. A randomized, placebocontrolled, double-blind, cross-over trial was conducted in 16 patients, simultaneous with their regular treatment (phototherapy, $n=$ 11 , and/or phenobarbital, $n=6$ ). Patients received orlistat or placebo, each for 4-6 wk. Compared with placebo, orlistat increased fecal fat excretion $(+333 \%)$ and fecal UCB excretion $(+43 \%)$. Orlistat treatment significantly decreased plasma UCB concentration $(-9 \%)$. In 7 of 16 patients, the decrease in plasma UCB levels was clinically relevant $(>10 \%$, mean $21 \%)$. In patients with a clinically relevant response, plasma UCB concentrations during orlistat were strongly, negatively correlated with fecal fat excretion $(r=-0.93)$. Clinically relevant response to orlistat treatment was not correlated with age, sex, CN type, BMI, or co-treatment with phototherapy or phenobarbital, but appeared correlated with a relatively lower dietary fat intake. In conclusion, orlistat treatment decreases plasma UCB concentrations, particularly in a subgroup of CN patients. Dietary fat intake may determine the responsiveness to orlistat treatment. (Pediatr Res 62: 725-730, 2007)
\end{abstract}

$\mathrm{C}$ rigler-Najjar $(\mathrm{CN})$ disease is a genetic disorder of bilirubin metabolism caused by deficiency of the hepatic enzyme bilirubin-UDP-glucuronosyltransferase (UGT1A1) (EC 2.4.1.17) (1). UGT1A1 catalyzes conjugation of unconjugated bilirubin (UCB), required for its efficient secretion into bile. CN patients suffer from permanent unconjugated hyperbilirubinemia which can cause bilirubin-induced neurologic damage (BIND) and kernicterus (2). The prevalence of CN disease is estimated at 1:1,000,000 (3). In The Netherlands, there are approximately 20 patients. In type-I CN disease, there is no detectable UGT1A1 activity. Plasma UCB concentrations in untreated type-I patients are above $350 \mu \mathrm{M}$ and can be as high as $800 \mu \mathrm{M}$, especially during intercurrent

Received March 26, 2007; accepted June 28, 2007.

Correspondence: H.J. Verkade, M.D., Ph.D., Department of Pediatrics, Pediatric Gastroenterology, University Medical Center Groningen, Room Y4.107a, P.O. Box 30.001, 9700 RB Groningen, The Netherlands; e-mail: h.j.verkade@med.umcg.nl Supported by a grant from the Najjar Foundation.

Based on this study, the Society for Pediatric Research awarded the 2007 SPR House Officer Research Award to AMH at the Pediatric Academic Societies' Meeting (Toronto, May 7, 2007). febrile illness (4). Plasma UCB levels in untreated type-II patients are generally below $350 \mu \mathrm{M}$ because of residual $( \pm 5 \%)$ enzyme activity, which can be enhanced by treatment with phenobarbital $(5,6)$.

To reduce the risk of kernicterus, type-I and some type-II patients need daily phototherapy. Phototherapy induces photoisomerization of UCB, which increases its hydrophilicity and biliary secretion (7). Phototherapy aims to keep plasma UCB levels below 350-400 $\mu \mathrm{M}$. Life-long daily phototherapy has considerable disadvantages. Main problems are a decreasing efficacy with age and a profound impact of the intensive phototherapy regimen on quality of (social) life $(4,8,9)$. At some stage, type-I patients usually have to undergo liver transplantation, which restores UGT1A1 activity, but has related morbidity and mortality and requires life-long immunosuppressive treatment (10). In the animal model for $\mathrm{CN}$ disease, the Gunn rat, gene therapy effectively restores UGT1A1 activity (11). However, vector toxicity and concerns about long-term safety have so far prevented the use of gene therapy in humans.

An alternative treatment option for unconjugated hyperbilirubinemia is based on intestinal capture of UCB. Particularly when plasma UCB concentrations are high as in $\mathrm{CN}$ disease, UCB can diffuse from the blood into the intestinal lumen across the mucosa $(12,13)$. Limited amounts of UCB are also secreted into bile $(14,15)$. Type-II patients secrete small amounts of bilirubin conjugates that are deconjugated to UCB after biliary secretion. Intestinal capture of UCB followed by fecal excretion reduces the enterohepatic circulation of UCB and subsequently decreases plasma UCB concentration.

Recently, we demonstrated in Gunn rats that orlistat treatment decreases plasma UCB concentrations parallel with increased fecal fat excretion, and induces net transmucosal excretion of UCB from blood into the intestinal lumen (1618). In human adults, orlistat has been widely applied for treatment of obesity, without serious side effects (19). Recent studies in obese adolescents and prepubertal children indicate that orlistat treatment is well-tolerated by children and generally only has mild side effects $(20,21)$. In the present study, we

Abbreviations: CN, Crigler-Najjar; UCB, unconjugated bilirubin; UGT1A1, bilirubin-UDP-glucuronosyltransferase 


\begin{tabular}{|c|c|c|c|c|c|c|c|c|c|c|c|c|c|c|}
\hline \multirow{2}{*}{$\begin{array}{l}\text { Period } \\
\text { Weeks }\end{array}$} & \multicolumn{2}{|c|}{ control } & \multicolumn{4}{|c|}{ orlistat or placebo } & \multicolumn{2}{|c|}{ control } & \multicolumn{4}{|c|}{ placebo or orlistat } & \multicolumn{2}{|c|}{ control } \\
\hline & -2 & -1 & 1 & 2 & 3 & 4 & 5 & 6 & 7 & 8 & 9 & 10 & 11 & 12 \\
\hline
\end{tabular}

Figure 1. Study design: placebo-controlled, cross-over trial. *Two weeks were added to this period of treatment. For study details, see "Methods."

determined the effects of orlistat treatment on plasma UCB concentrations in patients with $\mathrm{CN}$ disease.

\section{METHODS}

Study design. A randomized, placebo-controlled, double-blind, cross-over trial was conducted in $\mathrm{CN}$ patients (clinical trial registration number: NCT 00461799). Randomization was performed by a pharmacist of the Erasmus University Medical Center, Rotterdam. All patients started simultaneously with the trial to minimize environmental confounding factors such as variations in sunlight. The study was performed in the winter season. Current treatment with phototherapy $(n=11)$ and/or phenobarbital $(n=6)$ was continued during the trial, i.e. orlistat was tested as adjunct treatment. Patients received orlistat or placebo in a cross-over design with $2 \mathrm{wk}$ interval; each patient served as his/her own control (Fig. 1). Initial duration of the trial was $12 \mathrm{wk}$. The first 2 wk was a control period in which intra-individual variation in plasma bilirubin concentration was determined in three weekly samples. Orlistat or placebo would then be taken during $4 \mathrm{wk}$, followed by 2 -wk (wash-out) interval and then the alternate treatment during $4 \mathrm{wk}$. At the end of the study period, there was a posttreatment control period of $2 \mathrm{wk}$. Upon initiation of the trial, the dosage of the trial medication was adapted after $2 \mathrm{wk}$ (for details, see "Medication") and therefore $2 \mathrm{wk}$ were added to the trial, to allow comparison of treatment periods with similar dosages. Consequently, total duration of the trial was $14 \mathrm{wk}$.

Blood samples were taken weekly to determine plasma UCB concentration. Mean intra-individual variation in plasma UCB concentrations in the control period before start of treatment was $<10 \%$ (mean variation, $6 \pm 2 \%$ ). A clinically relevant response to orlistat treatment was therefore defined as a decrease in plasma UCB concentration of $\geq 10 \%$. Control UCB values were a mean of three weekly samples taken before start of orlistat. Response UCB values were a mean of two weekly samples taken at the end of orlistat treatment. Samples were taken at approximately the same hour each week to minimize diurnal variation of plasma UCB levels as confounding factor, and to ensure equal time periods between nightly phototherapy and blood sampling. Six times during the trial hematological and biochemical parameters were assessed (see "Analytical Methods"). In patients treated with phenobarbital, plasma levels were determined every 2 wk. Feces were collected 10 times at regular intervals during the trial. Three times, 72-h samples were collected to determine fecal fat excretion; once during each period (control, orlistat, placebo). The other seven times, a small sample was collected. Fecal fat and UCB concentrations were determined in all feces samples.

Patients. Sixteen patients participated in the trial; 13 from The Netherlands, 3 from Belgium. All patients, and/or their parents if the patient was younger than $18 \mathrm{y}$, gave written informed consent. The study protocol was approved by the Ethics Committee of the Erasmus University Medical Center, Rotterdam, The Netherlands, and of the Hôpital Universitaire des Enfants Reine Fabiola, Brussels, Belgium. To be included in the trial, patients had to be older than 7 y. Exclusion criteria were cholestasis, chronic malabsorption syndrome, and pregnancy. Stop criteria were defined as a patient's wish to stop or serious adverse events, defined as death, life-threatening events, hospitalization, or severe side effects (anaphylaxis, increase of liver transaminases $>30 \%$, increase of plasma UCB concentration $>50 \%$ with risk of kernicterus, plasma UCB level $>500 \mu \mathrm{M}$, severe coagulation problems, or diarrhea with $>10 \%$ weight loss). During the trial, body weight, blood pressure, and pulse rate were recorded weekly.

Medication. Orlistat (Xenical) is a selective inhibitor of gastrointestinal lipases that dose-dependently inhibits hydrolysis of dietary triglycerides (22). At a recommended dose of three times daily (t.i.d.) $120 \mathrm{mg}$, dietary fat absorption is reduced by approximately $30 \%$. Orlistat and placebo capsules were custom-made by the pharmacist of the Erasmus University Medical Center, Rotterdam. Initially, adults received $120 \mathrm{mg}$ t.i.d. during a meal, and children $66 \mathrm{mg} / \mathrm{m}^{2}$ body surface area t.i.d. (roughly equal the amount as an adult per square meter body surface area). However, after 2 wk of treatment, three patients suffered from side effects (diarrhea) and one patient (patient C, Table 1) from severely increased plasma UCB levels (maximum, $451 \mu \mathrm{M}$ ), after which the code was broken in close collaboration with the Ethics Committee. Symptoms appeared confined to orlistat-treated patients, after which the orlistat dosage was reduced by one third. This resulted in the following dosages: adults_-during breakfast and lunch $60 \mathrm{mg}$, during dinner $120 \mathrm{mg}$; children — during breakfast and lunch $33 \mathrm{mg} / \mathrm{m}^{2}$ body surface, during dinner $66 \mathrm{mg} / \mathrm{m}^{2}$ body surface area. The study protocol was prolonged by 2 wk, to allow patients to be compared for identical durations of treatment with the adapted orlistat dosage (i.e. 4 wk). The Ethics Committee approved the new study protocol. Cellulose was used as placebo. Previously, cellulose was shown to have no effect on plasma bilirubin concentrations (3).

Diet. Patients were instructed to eat their normal diet. Food intake was assessed three times during the trial, once during each period (control, orlistat, placebo). Patients and/or their parents were instructed by a dietician to keep a detailed record of the diet for three successive days, at the same days the 72-h feces samples were collected. Nutrient intake was calculated from a computerized Netherlands food composition database.

Compliance and phototherapy. Compliance was checked by counting remaining capsules afterward, by determination of fecal fat concentration, and by reviewing the diary patients had been instructed to keep. In this diary, patients recorded daily the times at which medication was taken, the number and consistency of bowel movements, and side effects, if any. Patients and/or their parents were instructed to use the phototherapy lights for approximately the same amount of hours every day and to keep a detailed record of

Table 1. Patient characteristics

\begin{tabular}{|c|c|c|c|c|c|c|c|c|}
\hline \multirow[b]{2}{*}{ Patient ID } & \multirow[b]{2}{*}{$\mathrm{CN}$ type } & \multirow[b]{2}{*}{ Age (y) } & \multirow[b]{2}{*}{$\operatorname{Sex}(\mathrm{M} / \mathrm{F})$} & \multirow[b]{2}{*}{ Body weight (kg) } & \multirow[b]{2}{*}{ BMI $\left(\mathrm{kg} / \mathrm{m}^{2}\right)$} & \multicolumn{2}{|c|}{$\begin{array}{c}\text { Phototherapy } \\
\text { ("special blue" bulbs) }\end{array}$} & \multirow[b]{2}{*}{ Phenobarbital (mg/d } \\
\hline & & & & & & (h/day) & No. bulbs/watt & \\
\hline B & I & 11 & F & 37 & 18 & $8-10$ & $12 / 100$ & - \\
\hline $\mathrm{C}$ & I & 14 & M & 56 & 19 & 11 & $10 / 100$ & - \\
\hline $\mathrm{D}$ & I & 14 & $\mathrm{~F}$ & 61 & 25 & $9-10$ & $12 / 100$ & - \\
\hline $\mathrm{G}$ & I & 19 & $\mathrm{~F}$ & 64 & 21 & 8 & $14 / 150$ & - \\
\hline $\mathrm{H}$ & II & 8 & $\mathrm{~F}$ & 29 & 16 & - & - & $2 \times 25$ \\
\hline I & II & 15 & M & 71 & 22 & 8 & $12 / 160$ & $1 \times 50,1 \times 75$ \\
\hline $\mathrm{J}$ & II & 17 & M & 56 & 18 & 1 & $20 / 100$ & $3 \times 50$ \\
\hline $\mathrm{K}$ & II & 27 & $\mathrm{~F}$ & 63 & 23 & - & - & Phenytoin $1 \times 200^{*}$ \\
\hline $\mathrm{L}^{\dagger}$ & II & 28 & $\mathrm{~F}$ & 85 & 25 & - & - & $1 \times 75,2 \times 50$ \\
\hline
\end{tabular}

* Phenytoin instead of phenobarbital. $\dagger$ Patient quit during the trial after 10 wk. 
Table 2. Hematological and biochemical parameters

\begin{tabular}{lcc}
\hline & Control & Orlistat \\
\hline Hemoglobin $(\mathrm{mmol} / \mathrm{L})$ & $8.8 \pm 0.6$ & $8.8 \pm 0.7$ \\
Hematocrit $(\mathrm{v} / \mathrm{v})$ & $0.41 \pm 0.03$ & $0.40 \pm 0.03$ \\
Reticulocytes $(\%)$ & $1.3 \pm 0.3$ & $1.3 \pm 0.3$ \\
ALT $(\mathrm{U} / \mathrm{l})$ & $67 \pm 69$ & $63 \pm 47$ \\
Albumin $(\mathrm{g} / \mathrm{L})$ & $45 \pm 2$ & $44 \pm 3$ \\
APTT $(\mathrm{sec})$ & $33 \pm 4$ & $34 \pm 4$ \\
PT $(\mathrm{sec})$ & $12 \pm 1$ & $12 \pm 1$ \\
Vitamin E (mmol/L) & $22 \pm 5$ & $18 \pm 4^{*}$ \\
\hline
\end{tabular}

$* p<0.001$

phototherapy times. All phototherapy units had "special blue" bulbs. We have determined that approximately every $1000 \mathrm{~h}$ these bulbs should be replaced. Light intensity levels were checked weekly with a Lux meter (measures light intensity in foot candles). To minimize the risk of confounding, we replaced half of the bulbs before and the other bulbs halfway during the study in the wash-out period. We estimate that maximum deterioration of the light levels would have been $10-20 \%$, if we had not replaced the bulbs.

Blood. Directly after vein puncture, tubes were protected from light and kept cool until analysis. All analytical procedures were performed in dim light. Plasma bilirubin levels protected from light, were analyzed in duplicate using the Hitachi-912-analyzer (Roche, Mannheim, Germany) and by reversed-phase HPLC, as described previously $(16,18)$. Other hematological and biochemical analyses were performed using routine clinical chemical procedures (Table 2).

Feces. Feces were stored in the dark at $-20^{\circ} \mathrm{C}$ until analysis. Fatty acids in feces were determined by gas chromatography (16). Fecal fat excretion was also determined via near-infrared assay (NIRA) of homogenized 72-h feces samples using a modification of the Van de Kamer method (23). Total fat analysis was performed by using the Soxtec Avanti-2050-system (FOSS Tecator AB, Hoganas, Sweden), which utilizes a four-step solvent (hexane) extraction technique. The process is fully automated, including hydrolysis, filtration, washing, and drying. Before performing the automated extraction method, $4 \mathrm{~g}$ of feces was dried overnight at $103^{\circ} \mathrm{C}$. UCB in feces was analyzed by reversed-phase HPLC, as described previously (18).

Statistical analyses. Power analysis was performed before start of the trial $[\alpha=0.05, \beta=0.20$, power $=0.80, \delta$ (clinically relevant response $)=0.10$ (10\%), numbers needed: $n=10-15](24,25)$. Statistical analyses were performed using SPSS 11.0 for Windows (SPSS Inc., Chicago, IL). Results are expressed as mean $\pm \mathrm{SD}$. Based on a normal distribution of plasma UCB levels in $\mathrm{CN}$ patients, parametric tests were used for statistical analysis. Intra-individual differences were tested with the paired $t$ test. The relationship between plasma UCB and fecal fat was analyzed by linear regression analysis. Correlation analysis was done with Pearson's cross-tables. $p$ Values $<0.05$ (two-tailed) were considered significant.

\section{RESULTS}

Patient characteristics. Sixteen patients with CN disease were included (7 type-I, 9 type-II, Table 1). Median age was 17.5 y (range, $8-51$ y). Male to female ratio was 5:11 (31\%:69\%). Median body weight was $64 \mathrm{~kg}$ (range, 29-85 $\mathrm{kg}$ ). Median BMI was 22.5 (range, 16-30). Eleven patients received phototherapy treatment. The daily amount of hours of phototherapy ranged from an average of $9.4 \mathrm{~h}$ in type-I patients to $1-2 \mathrm{~h}$ in some type-II patients. Five type-II patients were treated with phenobarbital and one with phenytoin.

General effects of orlistat. Orlistat treatment did not significantly affect body weight (average body weight without orlistat, $64 \pm 16 \mathrm{~kg}$, versus orlistat, $64 \pm 17 \mathrm{~kg}, \mathrm{NS}$ ). $\mathrm{Hb}$, hematocrit, and reticulocyte count were not affected by orlistat treatment, and neither were plasma albumin concentrations, ALT, APTT, and PT (Table 2). Orlistat treatment significantly decreased vitamin E levels $(-18 \%, p<$ 0.001). In patients treated with phenobarbital, plasma levels were stable during the trial based on sampling every 2
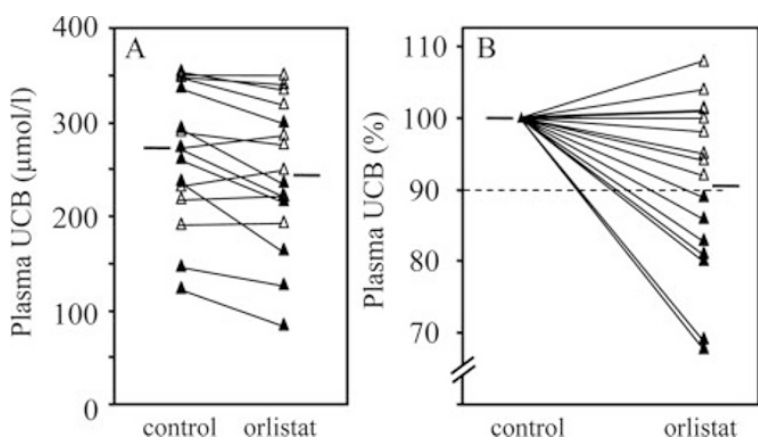

Figure 2. Effects of orlistat treatment on plasma UCB concentrations in $\mathrm{CN}$ patients. Each set of triangles represents data from one patient. Response to orlistat treatment was defined as a decrease in plasma UCB concentration of at least $10 \%$. Responders $(\mathbf{\Lambda})$, nonresponders $(\Delta)$. For study details, see "Methods."

weeks (average levels without orlistat, $13.0 \pm 3.5 \mathrm{mg} / \mathrm{L}$, versus orlistat, $12.5 \pm 4.0 \mathrm{mg} / \mathrm{L}, \mathrm{NS}$ ).

Effects of orlistat on plasma UCB concentrations. Before orlistat treatment, plasma UCB concentrations ranged from 123 to $354 \mu \mathrm{M}$ (type-I patients, $317 \pm 36 \mu \mathrm{M}$; type-II patients, $228 \pm 71 \mu \mathrm{M}$; Fig. $2 A$ ). Within each patient, plasma UCB levels were fairly constant (mean variation $6 \pm 2 \%$ ). Orlistat treatment decreased plasma UCB concentrations by $9 \pm 12 \%(p<0.01$; range, -32 to $+8 \%$; Fig. $2 B$ ). Based on the definition of a clinically relevant response to treatment, 7 of the 16 patients $(44 \%)$ responded, with a mean decrease of $21 \%$ in plasma UCB levels (range, 11-32\%).

Effects of orlistat on fecal fat and UCB excretion. Orlistat treatment increased fecal fat concentration in all patients (from $0.08 \pm 0.03$ to $0.31 \pm 0.10 \mathrm{mmol} / \mathrm{g}$ feces, $+333 \pm$ $249 \%$, according to gas chromatographic analysis of fecal fatty acids; $p<0.001$; data not shown), and total fecal fat excretion (from $3.0 \pm 1.7$ to $16.8 \pm 8.1 \mathrm{~g} / 24 \mathrm{~h},+652 \pm$ $516 \%$, according to the NIRA method of fecal fat analysis; $p<0.001$; Fig. 3). Fecal fat concentration and excretion were not different between orlistat responsive and nonresponsive patients. Orlistat treatment also increased fecal concentration of UCB $(+43 \%, p<0.05$; range, -46 to $+223 \%$; Fig. 4). Under control conditions, fecal UCB concentrations varied between patients from 0.05 to $0.31 \mu \mathrm{mol} / \mathrm{g}$ feces (mean $0.14 \pm 0.08)$. Fecal UCB concentrations did not

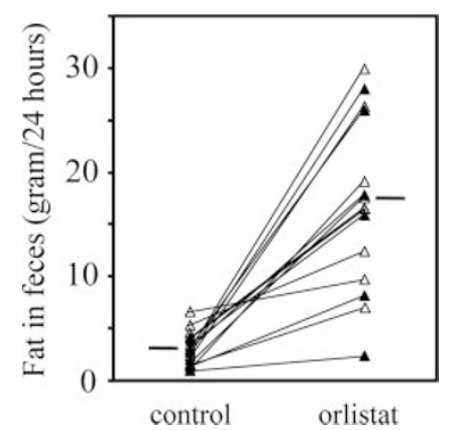

Figure 3. Effects of orlistat treatment on fecal fat excretion in CN-patients. Each set of triangles represents data from one patient. Responders $(\mathbf{\Delta})$, nonresponders $(\Delta)$. For study details, see "Methods." 

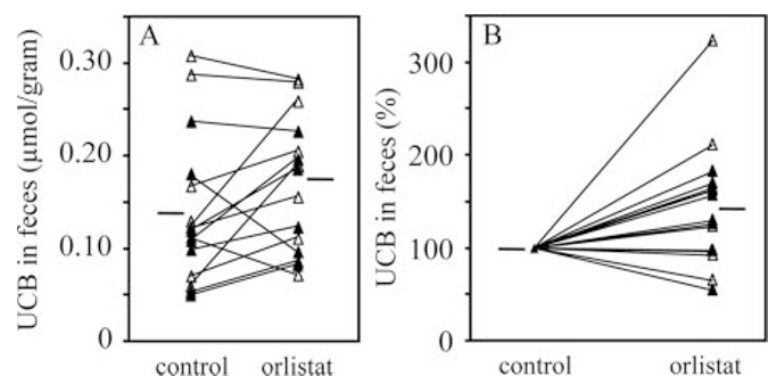

Figure 4. Effects of orlistat treatment on fecal UCB excretion in CN-patients. Each set of triangles represents data from one patient. Responders $(\mathbf{\Lambda})$, nonresponders $(\Delta)$. For study details, see "Methods."
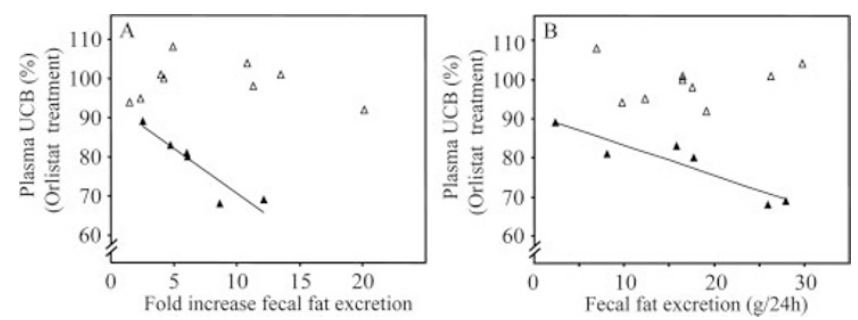

Figure 5. Relationship between plasma UCB concentration and fecal fat excretion during orlistat treatment in $\mathrm{CN}$ patients. A negative linear correlation was observed in patients who responded to orlistat treatment $(r=-0.93$, $p<0.01)$. Each triangle represents data from one patient. Responders $(\mathbf{\Delta})$, nonresponders $(\Delta)$. For study details, see "Methods."

differ significantly between orlistat-responsive and nonresponsive patients, either under control conditions or during orlistat treatment.

In previous studies in Gunn rats, the change in plasma UCB concentration was strongly correlated with the amount of fat excreted via the feces $(16,18)$. Figure 5 shows the relationship between parameters of fecal fat excretion and the orlistatinduced alteration in plasma UCB concentration. Plasma UCB concentrations in responding $\mathrm{CN}$ patients were strongly, negatively related to parameters of fecal fat excretion $(r=-0.93$, $p<0.01$, Fig. 5). In contrast, plasma UCB concentrations in nonresponding patients were independent of fecal fat excretion. Net fat uptake, calculated as the difference between total fat intake and fecal fat excretion, tended to be lower in

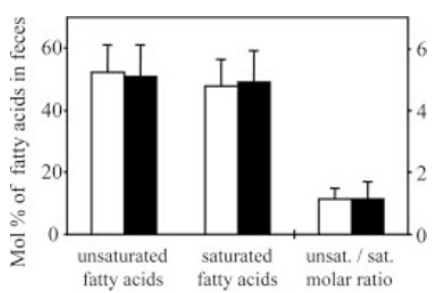

Figure 6. Molar fractions of saturated and unsaturated fatty acids in feces of $\mathrm{CN}$ patients during orlistat treatment. Differences were not statistically significant. Responders ( $\square$ ), nonresponders $(\square)$. For study details, see "Methods."

responders compared with nonresponders $(58 \pm 31$ and $86 \pm$ $31 \mathrm{~g} / 24 \mathrm{~h}$, respectively, $p=0.11$ ).

Characteristics of responsive versus nonresponsive patients. Responders and nonresponders did not differ significantly in the amount of saturated or unsaturated fatty acids excreted via the feces, nor in their relative (molar) ratio in feces (Fig. 6). Accordingly, neither saturated nor unsaturated fecal fat appeared specifically related to plasma UCB concentrations. In a study in which $\mathrm{CN}$ patients were treated with calcium phosphate, differences in response to treatment correlated with the type of $\mathrm{CN}$ disease and with co-treatment with phototherapy (3). Since our data also indicated differences in responsiveness to orlistat treatment, we analyzed whether these or other patient characteristics correlated with (non)responsiveness (Table 3). (Non)responsiveness of plasma UCB concentrations to orlistat treatment did not significantly correlate with either $\mathrm{CN}$ type, age, sex, body weight, or cotreatment with phototherapy or phenobarbital. Interestingly, however, the majority of responsive patients had a total dietary fat intake $<35$ energy $\%(p=0.05)$, and all responsive patients had a body mass index (BMI) $\leq 25(p=0.10)$. Dietary intake of saturated fat, cholesterol, calcium, or fiber was not significantly different between orlistat responsive and nonresponsive patients.

Side effects and compliance. As mentioned in "Methods," after $2 \mathrm{wk}$ of treatment, the orlistat dosage had to be adapted because of side effects. Side effects were almost exclusively related to the gastrointestinal tract, such as diarrhea, oily leakage, stomach cramps, and flatulence. After dosage reduc-

Table 3. Correlations (non)response vs patient characteristics/dietary intake

\begin{tabular}{|c|c|c|c|c|}
\hline \multicolumn{2}{|c|}{ Patient characteristics } & \multirow{2}{*}{$\frac{\text { Nonresponders }(n=9)}{44 \%}$} & \multirow{2}{*}{$\frac{\text { Responders }(n=7)}{43 \%}$} & \multirow{2}{*}{$\frac{p \text { Value }}{0.95}$} \\
\hline $\mathrm{CN}$ type & I & & & \\
\hline Age & $<18 \mathrm{y}$ & $44 \%$ & $57 \%$ & 0.64 \\
\hline Sex & Female & $56 \%$ & $86 \%$ & 0.22 \\
\hline Phototherapy & Yes & $78 \%$ & $57 \%$ & 0.41 \\
\hline Phenobarbital* & Yes & $33 \%$ & $43 \%$ & 0.72 \\
\hline Total fat & $<35$ energy $\%$ & $22 \%$ & $71 \%$ & 0.05 \\
\hline Saturated fat & $<13$ energy $\%$ & $33 \%$ & $43 \%$ & 0.72 \\
\hline Cholesterol & $<220 \mathrm{mg}$ & $33 \%$ & $71 \%$ & 0.15 \\
\hline Calcium & $<800 \mathrm{mg}$ & $67 \%$ & $71 \%$ & 0.85 \\
\hline Fiber & $<17 \mathrm{~g}$ & $67 \%$ & $86 \%$ & 0.42 \\
\hline
\end{tabular}

* In one patient phenytoin in stead of phenobarbital 
tion side effects were generally mild, temporary, and tolerable for all patients. Oily leakage and diarrhea were remarkably yellow/orange colored. Mild side effects such as greasy appearance of the feces occurred in almost all patients. Some side effects (such as flatulence) were also reported during placebo treatment. Headache was incidentally reported, equally during placebo and orlistat treatment. Five patients reported improved well-being, and felt more energetic during orlistat treatment. All these patients would have liked to continue orlistat treatment after the trial. These subjective effects did not correlate with responsiveness. Two of these five patients responded to orlistat treatment (plasma UCB concentrations decreased by $31 \%$ and $20 \%$ ), but in the other three patients plasma UCB concentrations did not decrease to a clinically relevant extent $(<10 \%)$. Three patients reported increased appetite during orlistat. As stated, one patient (patient $\mathrm{C}$, Table 1) stopped during $1 \mathrm{wk}$ with orlistat treatment because of an increase in plasma UCB levels after 1 wk of treatment. After dosage reduction, the treatment was restarted and an extra week was added to the treatment period of this patient. Another patient (patient L, Table 1) dropped out from the trial at $10 \mathrm{wk}$, because of psychological issues (e.g. lack of concentration, mood swings). Compliance to medication appeared to be good in all patients, based on capsule counting, corresponding diary entries, and, by inference, from increased fecal fat excretion during orlistat treatment. With regard to phototherapy, patients received the same number of hours of phototherapy during control periods and placebo or orlistat treatment. Light levels checked weekly with a Lux meter, showed that lamps deteriorated during the study period by approximately $10 \%$.

\section{DISCUSSION}

Orlistat treatment decreased plasma UCB concentrations. The decrease was statistically significant, however, it was only clinically relevant (more than 10\% decrease) in approximately $40 \%$ of the patients. In this orlistat-responsive subgroup, the decrease in plasma UCB concentration was strongly related to orlistat-induced fecal fat excretion. Responsiveness of $\mathrm{CN}$ patients could not be predicted by patient characteristics, except that responders tended to have a lower dietary fat intake and lower BMI.

Responsiveness to orlistat treatment was independent of co-treatment with phototherapy or phenobarbital, CN type, body weight, sex, or age. However, the subgroup of patients that responded to orlistat treatment tended to have a lower dietary fat intake and lower BMI than nonresponsive patients. This observation corresponds with our previous results in Gunn rats, in which orlistat treatment was more effective when rats were fed a low-fat compared with a high-fat diet (16). Possibly, patients with a high fat intake (and consequently higher fecal fat excretion) have already reached a maximum level of UCB capture by fat and increasing fat intake and/or excretion may therefore not (further) decrease plasma UCB concentrations. The subgroup of (clinically relevant) responders is specific in one other phenomenon. Only in this subgroup, plasma UCB levels were strongly, negatively corre- lated with fecal fat excretion. Previously, we showed a similar relationship between plasma UCB concentration and fecal fat excretion in Gunn rats (18). The hypobilirubinemic effect of orlistat in Gunn rats is partially due to increased metabolism of UCB to derivatives (17). Others have also shown the importance of the intestinal microflora for UCB metabolism (26). We speculate that the orlistat effect on UCB is predominantly associated with the excretion of fecal fatty acids or partly digested triacylglycerols, rather than with fecal triglyceride excretion. Possibly, the orlistat dose has to be individualized to create a certain amount of lipase inhibition/fat malabsorption with a certain optimal distribution of fatty acids or partially hydrolyzed triglycerides in the intestinal lumen. This would explain why in one patient, plasma UCB levels increased after $1 \mathrm{wk}$ of treatment with orlistat. In his feces, the percentage of triglycerides relative to FFA was increased more than in other patients. His plasma UCB levels decreased after orlistat dosis reduction.

Orlistat treatment increased fecal UCB concentration by $43 \%$. Presumably, the primary effect of orlistat treatment is intestinal capture of UCB by fat. In some responsive patients, fecal UCB concentrations clearly increased during orlistat treatment. However, fecal UCB concentration also increased in some patients in which plasma UCB levels did not decrease significantly during orlistat treatment. Differences in dietary (fat) intake, composition or metabolic activity of the intestinal microflora, lipase levels, and steady state of the UCB- and derivatives pool may account for these differences in this small group of patients.

The number of $\mathrm{CN}$ patients that could participate in the trial was inevitably limited due to low prevalence of the disease. To overcome this, a cross-over study design was chosen. We obtained indications that the duration of treatment and washout period may have been relatively short, and could have resulted in underestimation of the orlistat effect. Firstly, the majority of patients had an increased fecal UCB content at the end of orlistat treatment, suggesting that they had not yet reached a steady-state in UCB homeostasis. Secondly, in the first week of the wash-out period, plasma UCB concentrations did not readily return to pretreatment values. The hypobilirubinemic effects of orlistat may be more pronounced upon prolonged treatment.

Orlistat treatment, at the eventual dose used, did not cause major side effects. The generally mild, temporary and tolerable side effects that did occur were similar to those reported in trials where orlistat was used for treatment of obesity $(19,21)$. Side effects were almost exclusively related to the gastrointestinal tract. Orlistat treatment decreased plasma vitamin E levels, as previously described by others (19). Low vitamin levels due to prolonged orlistat treatment could easily be prevented by dietary supplementation. We do not know what the impact of orlistat treatment on the daily lives of $\mathrm{CN}$ patients would be. Only in patients with a significant decrease in plasma UCB levels, we expect it would reduce the amount of phototherapy needed, which would most likely improve quality of (social) life. At present, orlistat cannot be uniformly recommended as adjunct treatment for $\mathrm{CN}$ disease. 
In conclusion, present data indicate that orlistat can be useful for treatment of unconjugated hyperbilirubinemia, particularly in a subgroup of $\mathrm{CN}$ patients. Dietary fat intake may determine responsiveness to orlistat treatment. It will be a challenge for future research to identify other relevant parameters or patient characteristics that could predict responsiveness of $\mathrm{CN}$ patients to orlistat treatment. In addition, it needs to be addressed whether prolonged orlistat treatment could safely and reliably decrease the need for phototherapy.

Acknowledgments. The authors thank Dr. J.H.P. Wilson, Dr. P. Goyens, M. van Eygen, L.G. Vanbecelaere, A.D. Lindemans and T. van der Zanden for their assistance throughout the trial, M. Verhagen for analyzing dietary nutrient intake, and Dr. Y.B. de Rijke for fecal fat analysis.

\section{REFERENCES}

1. Crigler JF, Najjar VA 1952 Congenital familial nonhemolytic jaundice with kernicterus. Pediatrics 10:169-180

2. Shapiro SM 2005 Definition of the clinical spectrum of kernicterus and bilirubininduced neurologic dysfunction (BIND). J Perinatol 25:54-59

3. Van der Veere CN, Jansen PL, Sinaasappel M, Van der Meer R, Van der Sijs H, Rammeloo JA, Goyens P, Van Nieuwkerk CM, Oude Elferink RP 1997 Oral calcium phosphate: a new therapy for Crigler-Najjar disease? Gastroenterology 112:455-462

4. Van der Veere CN, Sinaasappel M, McDonagh AF, Rosenthal P, Labrune P, Odievre M, Fevery J, Otte JB, McClean P, Burk G, Masakowski V, Sperl W, Mowat AP, Vergani GM, Heller K, Wilson JP, Shepherd R, Jansen PL 1996 Current therapy for Crigler-Najjar syndrome type 1: report of a world registry. Hepatology 24:311-315

5. Catz C, Yaffe SJ 1968 Barbiturate enhancement of bilirubin conjugation and excretion in young and adult animals. Pediatr Res 2:361-370

6. Yaffe SJ, Levy G, Matsuzawa T, Baliah T 1966 Enhancement of glucuronideconjugating capacity in a hyperbilirubinemic infant due to apparent enzyme induction by phenobarbital. N Engl J Med 275:1461-1466

7. Ostrow JD 1971 Photocatabolism of labeled bilirubin in the congenitally jaundiced (Gunn) rat. J Clin Invest 50:707-718

8. Strauss KA, Robinson DL, Vreman HJ, Puffenberger EG, Hart G, Morton DH 2006 Management of hyperbilirubinemia and prevention of kernicterus in 20 patients with Crigler-Najjar disease. Eur J Pediatr 165:306-319
9. Yohannan MD, Terry HJ, Littlewood JM 1983 Long term phototherapy in CriglerNajjar syndrome. Arch Dis Child 58:460-462

10. Whitington PF, Emond JC, Heffron T, Thistlethwaite JR 1993 Orthotopic auxiliary liver transplantation for Crigler-Najjar syndrome type 1. Lancet 342 : 779-780

11. Toietta G, Mane VP, Norona WS, Finegold MJ, Ng P, McDonagh AF, Beaudet AL, Lee B 2005 Lifelong elimination of hyperbilirubinemia in the Gunn rat with a single injection of helper-dependent adenoviral vector. Proc Natl Acad Sci U S A 102:3930-3935

12. Kotal P, Van Der Veere CN, Sinaasappel M, Elferink RO, Vitek L, Brodanova M, Jansen PL, Fevery J 1997 Intestinal excretion of unconjugated bilirubin in man and rats with inherited unconjugated hyperbilirubinemia. Pediatr Res 42:195200

13. Schmid R, Hammaker L 1963 Metabolism and disposition of C14-bilirubin in congenital nonhemolytic jaundice. J Clin Invest 42:1720-1734

14. Clarenburg R, Kao CC 1973 Shared and separate pathways for biliary excretion of bilirubin and BSP in rats. Am J Physiol 225:192-200

15. Chowdhury JR, Wolkoff AW, Roy Chowdhury >NR, Arias IM. 2001 Hereditary jaundice and disorders of bilirubin metabolism. In: Scriver CR, Beaudet AL, Sly WS, Valle D (eds) The Metabolic and Molecular Bases of Inherited Disease. Vol. McGraw-Hill, New York, pp 2161-2208

16. Hafkamp AM, Havinga R, Sinaasappel M, Verkade HJ 2005 Effective oral treatment of unconjugated hyperbilirubinemia in Gunn rats. Hepatology 41:526-534

17. Hafkamp AM, Havinga R, Ostrow JD, Tiribelli C, Pascolo L, Sinaasappel M, Verkade HJ 2006 Novel kinetic insights into treatment of unconjugated hyperbilirubinemia: phototherapy and orlistat treatment in Gunn rats. Pediatr Res 59:506512

18. Nishioka T, Hafkamp AM, Havinga R, van Lierop PP, Velvis H, Verkade HJ 2003 Orlistat treatment increases fecal bilirubin excretion and decreases plasma bilirubin concentrations in hyperbilirubinemic Gunn rats. J Pediatr 143:327-334

19. Ballinger A, Peikin SR 2002 Orlistat: its current status as an anti-obesity drug. Eur J Pharmacol 440:109-117

20. Norgren S, Danielsson P, Jurold R, Lotborn M, Marcus C 2003 Orlistat treatment in obese prepubertal children: a pilot study. Acta Paediatr 92:666-670

21. Chanoine JP, Hampl S, Jensen C, Boldrin M, Hauptman J 2005 Effect of orlistat on weight and body composition in obese adolescents: a randomized controlled trial. JAMA 293:2873-2883

22. Guerciolini R 1997 Mode of action of orlistat. Int J Obes Relat Metab Disord 21:S12-S23

23. Van de Kamer JH 1953 Quantitative determination of the saturated and unsaturated higher fatty acids in fecal fat. Scand J Clin Lab Invest 5:30-36

24. Altman DG 1991 Practical Statistics for Medical Research. Chapman \& Hall/CRC, London, pp 455-460

25. Pocock SJ 1983 Clinical Trials: A Practical Approach. Wiley \& Sons, New York, pp $123-130$

26. Vitek L, Zelenka J, Zadinova M, Malina J 2005 The impact of intestinal microflora on serum bilirubin levels. J Hepatol 42:238-243 